Supplementary Materials for

\title{
Deep Oxidative Desulfurization of Model Fuels Catalyzed by Subnanosized Ti Oxoclusters
}

\author{
Tong Zhang ${ }^{\dagger}$ Xuejing Chen ${ }^{\dagger}$, Bingjie Ding ${ }^{\dagger}$ Zuoji Ding ${ }^{\dagger}$, Yajun Wang ${ }^{\dagger}$, Hewen Qiu \\ Yongjun Jiang $\$$, Sheng Dai*§, Zhenshan Hou ${ }^{\dagger}$;*
}

${ }^{\dagger}$ Key Laboratory for Advanced Materials, Research Institute of Industrial Catalysis, School of Chemistry and Molecular Engineering, East China University of Science and Technology, Shanghai 200237, China.

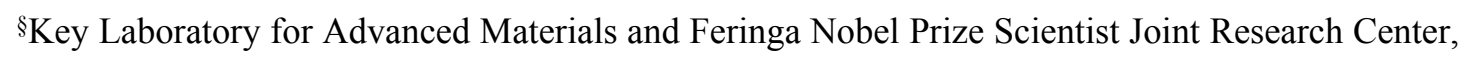
Institute of Fine Chemicals, School of Chemistry \& Molecular Engineering, East China University of Science and Technology, Shanghai 200237, P. R. China.

Shanghai Key Laboratory of Green Chemistry and Chemical Processes, East China Normal University, School of Chemistry and Molecular Engineering, Shanghai 200062, China.

*Corresponding author.

E-mail: houzhenshan@ecust.edu.cn

E-mail: shengdai@ecust.edu.cn

\section{This file includes:}

Scheme S1. Synthesis of carboxylic acid functionalized imidazolium based-ILs.

Figure S1. ${ }^{1} \mathrm{H}$ NMR and ${ }^{13} \mathrm{C}$ NMR spectra of $\left[\mathrm{C}_{4} \mathrm{IMC}_{2} \mathrm{COOH}\right] \mathrm{Br}$.

Figure S2. ${ }^{1} \mathrm{H}$ NMR and ${ }^{13} \mathrm{C}$ NMR spectra of $\left[\mathrm{C}_{4} \mathrm{IMC}_{2} \mathrm{COOH}\right]\left[\mathrm{HSO}_{4}\right]$.

Figure S3. ${ }^{1} \mathrm{H}$ NMR and ${ }^{13} \mathrm{C}$ NMR spectra of $\left[\mathrm{C}_{4} \mathrm{IMC}_{2} \mathrm{COOH}\right][\mathrm{OTf}]$.

Figure S4. ${ }^{1} \mathrm{H}$ NMR and ${ }^{13} \mathrm{C}$ NMR spectra of $\left[\mathrm{C}_{4} \mathrm{IMC}_{2} \mathrm{COOH}\right]\left[\mathrm{PF}_{6}\right]$.

Figure S5. ${ }^{1} \mathrm{H}$ NMR and ${ }^{13} \mathrm{C}$ NMR spectra of $[\mathrm{BMIM}]\left[\mathrm{HSO}_{4}\right]$.

Figure S6. XRD patterns of 500- $\mathrm{TiO}_{2}$ and different IL-stabilized Ti oxoclusters.

Figure S7. TGA patterns of different Ti oxoclusters.

Figure S8. HRTEM images of 500- $\mathrm{TiO}_{2}$ with different magnification.

Figure S9 HRTEM image of Ti oxo-[BMIM] $\mathrm{HSO}_{4}$.

Figure S10. Removal of DBT versus the reaction time with different volume ratios of oil to $\mathrm{MeOH}$ in ECODS process. 
Figure S11. (A) The photograph of the IL-stabilized Ti oxoclusters and $500-\mathrm{TiO}_{2}$ catalysts in methanol/model oil of DBT biphasic systems; (B) After $\mathrm{H}_{2} \mathrm{O}_{2}$ was introduced into the mixture to form ECODS system; (C) Reaction for $30 \mathrm{~min}$.

Figure S12. Removal of DBT versus the reaction time with different amounts of Ti oxo$\mathrm{HSO}_{4}$.

Figure S13. FT-IR spectra of the spent Ti oxo- $\mathrm{HSO}_{4}$ catalyst (without dryness) after six catalytic recycles.

Figure S14 Reusability of Ti oxo-[BMIM] $\mathrm{HSO}_{4}$.

Figure S15. Effect of temperature on sulfur removal of actual fuel samples.

Figure $\mathrm{S} 16$. The relationship between the reaction rate $\left(\mathrm{R}_{0}\right)$ and the concentration of $\operatorname{DBT}(a, b)$.

Table S1. Comparison of catalytic systems for oxidative desulfurization of DBT in the presence of $\mathrm{H}_{2} \mathrm{O}_{2}$.

Figure S17. (Row 1, Black line) DRUV-Vis spectra of Ti oxoclusters; (Row 1, Red line) DRUV-Vis spectra of $\mathrm{Ti}$ oxoclusters treated with $\mathrm{H}_{2} \mathrm{O}_{2}$; (Row 2) The corresponding deconvoluted spectra of Ti oxoclusters treated with $\mathrm{H}_{2} \mathrm{O}_{2}$.

Figure S18. FT-IR spectra of Ti oxoclusters treated with $\mathrm{H}_{2} \mathrm{O}_{2}$. 


\section{Preparation of the functionalized imidazolium based-Ionic liquids (ILs)}

The ILs have been synthesized by following approach in this work (Scheme S1).

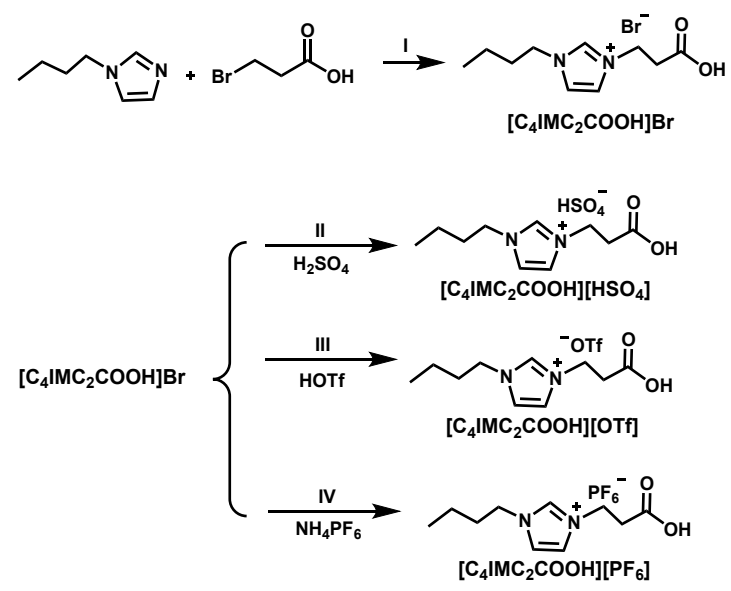

Scheme S1 Synthesis of carboxylic acid functionalized imidazolium based-ILs. Reagents and reaction conditions: (I) 3-bromopropionic acid (1.1 equivalent), acetonitrile, reflux at $80{ }^{\circ} \mathrm{C}, 24 \mathrm{~h}$; (II) $\mathrm{H}_{2} \mathrm{SO}_{4}$ (1 equivalent), $\mathrm{MeOH}, 65{ }^{\circ} \mathrm{C}$ then reflux, $24 \mathrm{~h}$; (III) HOTf (1 equivalent), $\mathrm{MeOH}, 65{ }^{\circ} \mathrm{C}$ then reflux, $24 \mathrm{~h}$; (IV) $\mathrm{NH}_{4} \mathrm{PF}_{6}$ (1 equivalent), deionized water, r.t., $2 \mathrm{~h}$.

\section{1-(2-Carboxyethyl)-3-butylimidazolium bromide}

1-(2-Carboxyethyl)-3-butylimidazolium bromide $\left(\left[\mathrm{C}_{4} \mathrm{IMC}_{2} \mathrm{COOH}\right] \mathrm{Br}\right)$ was prepared by an one-step method. ${ }^{1}$ Under nitrogen atmosphere, 3-bromopropionic acid ( $1.6827 \mathrm{~g}, 11 \mathrm{mmol})$ was added into a $50 \mathrm{~mL}$ Schlenk bottle at room temperature and dissolved in acetonitrile $(10 \mathrm{~mL})$. N-butyl imidazole $(1.2418 \mathrm{~g}, 10 \mathrm{mmol})$ was added dropwise to the above solution. After the addition was completed, the entire reaction system was heated to $80^{\circ} \mathrm{C}$ and refluxed for $24 \mathrm{~h}$, and the color of the solution changed from colorless to light yellow. After the reaction, the acetonitrile was removed by rotary evaporation to obtain a crude IL, which was washed with ethyl acetate/diethyl ether three times to remove unreacted 3-bromopropionic acid and other impurities. The product was then dried under vacuum at $60{ }^{\circ} \mathrm{C}$ for $12 \mathrm{~h}$ to afford a light brown and viscous liquid. Yield: $2.31 \mathrm{~g}(83.3 \%)$. $\left[\mathrm{C}_{4} \mathrm{IMC}_{2} \mathrm{COOH}\right] \mathrm{Br}:{ }^{1} \mathrm{H} \mathrm{NMR}\left(400 \mathrm{MHz}, \mathrm{D}_{2} \mathrm{O}\right.$, ppm): $\delta 8.70(\mathrm{~s}, 1 \mathrm{H}), 7.40(\mathrm{~s}, 1 \mathrm{H}), 7.36(\mathrm{~s}, 1 \mathrm{H}), 4.33(\mathrm{t}, 2 \mathrm{H}), 4.04(\mathrm{t}, 2 \mathrm{H}), 2.87(\mathrm{t}, 2 \mathrm{H})$, 
$1.70(\mathrm{~m}, 2 \mathrm{H}), 1.14(\mathrm{~m}, 2 \mathrm{H}), 0.77(\mathrm{t}, 3 \mathrm{H}) ;{ }^{13} \mathrm{C}$ NMR (100 MHz, $\left.\mathrm{D}_{2} \mathrm{O}, \mathrm{ppm}\right): \delta 174.29$, 135.71, 122.29, 121.71, 49.39, 44.96, 33.75, 31.23, 18.97, 12.29 (Fig. S1).

\section{1-(2-Carboxyethyl)-3-butylimidazolium hydrogen sulfate}

1-(2-Carboxyethyl)-3-butylimidazolium hydrogen sulfate $\left(\left[\mathrm{C}_{4} \mathrm{IMC}_{2} \mathrm{COOH}\right]\left[\mathrm{HSO}_{4}\right]\right)$ was prepared by a two-step method. In the first step, $\left[\mathrm{C}_{4} \mathrm{IMC}_{2} \mathrm{COOH}\right] \mathrm{Br}$ was prepared according to the above method. In the second step, under nitrogen atmosphere, $\left[\mathrm{C}_{4} \mathrm{IMC}_{2} \mathrm{COOH}\right] \mathrm{Br}$ was added $(1.3858 \mathrm{~g}, 5 \mathrm{mmol})$ into a $50 \mathrm{~mL}$ Schlenk bottle and dissolved in methanol $(10 \mathrm{~mL})$. Then an equivalent amount of concentrated $\mathrm{H}_{2} \mathrm{SO}_{4}(97 \%, 0.5056 \mathrm{~g}, 5 \mathrm{mmol})$ was added dropwise to the above solution. Afterwards, the reaction mixture was heated to $65^{\circ} \mathrm{C}$ and refluxed for $24 \mathrm{~h}$. After the reaction, the methanol was removed by rotary evaporation, then the $\mathrm{HBr}$ generated in the reaction was removed using a vacuum cryogenic device (liquid nitrogen). The IL was further dried at $60{ }^{\circ} \mathrm{C}$ for $12 \mathrm{~h}$. The IL was obtained as a light yellow viscous liquid. Yield: $1.28 \mathrm{~g}(87.1 \%)$. [C $\left.\mathrm{C}_{4} \mathrm{IMC}_{2} \mathrm{COOH}\right]\left[\mathrm{HSO}_{4}\right]:{ }^{1} \mathrm{H}$ NMR (400 $\left.\mathrm{MHz}, \mathrm{D}_{2} \mathrm{O}, \mathrm{ppm}\right): \delta 8.70(\mathrm{~s}, 1 \mathrm{H}), 7.40(\mathrm{~s}, 1 \mathrm{H}), 7.35(\mathrm{~s}, 1 \mathrm{H}), 4.34(\mathrm{t}, 2 \mathrm{H}), 4.06(\mathrm{t}, 2 \mathrm{H})$, $2.87(\mathrm{t}, 2 \mathrm{H}), 1.70(\mathrm{~m}, 2 \mathrm{H}), 1.16(\mathrm{~m}, 2 \mathrm{H}), 0.77(\mathrm{t}, 3 \mathrm{H}) ;{ }^{13} \mathrm{C} \mathrm{NMR}\left(100 \mathrm{MHz}, \mathrm{D}_{2} \mathrm{O}, \mathrm{ppm}\right)$ : $\delta 172.84,135.71,122.59,121.72,49.10,44.70,34.06,30.97,18.98,12.29$ (Fig. S2).

\section{1-(2-Carboxyethyl)-3-butylimidazolium trifluoromethanesulfonate}

The synthetic route of 1-(2-carboxyethyl)-3-butylimidazolium trifluoromethanesulfonate $\left(\left[\mathrm{C}_{4} \mathrm{IMC}_{2} \mathrm{COOH}\right][\mathrm{OTf}]\right)$ was the same as that of $\left[\mathrm{C}_{4} \mathrm{IMC}_{2} \mathrm{COOH}\right]\left[\mathrm{HSO}_{4}\right]$, except that the concentrated $\mathrm{H}_{2} \mathrm{SO}_{4}$ was replaced by HOTf (0.7504g, $5 \mathrm{mmol})$. The product was a brown viscous liquid. Yield: $\sim 1.52 \mathrm{~g}(87.8 \%)$. $\left[\mathrm{C}_{4} \mathrm{IMC}_{2} \mathrm{COOH}\right][\mathrm{OTf}]:{ }^{1} \mathrm{H}$ NMR $\left(400 \mathrm{MHz}, \mathrm{D}_{2} \mathrm{O}, \mathrm{ppm}\right): \delta 8.66(\mathrm{~s}, 1 \mathrm{H}), 7.43(\mathrm{~s}, 1 \mathrm{H})$, $7.38(\mathrm{~s}, 1 \mathrm{H}), 4.39(\mathrm{t}, 2 \mathrm{H}), 4.13(\mathrm{t}, 2 \mathrm{H}), 2.88(\mathrm{t}, 2 \mathrm{H}), 1.75(\mathrm{~m}, 2 \mathrm{H}), 1.20(\mathrm{~m}, 2 \mathrm{H}), 0.81(\mathrm{t}$, $3 \mathrm{H}) ;{ }^{13} \mathrm{C}$ NMR (100 MHz, $\left.\mathrm{D}_{2} \mathrm{O}, \mathrm{ppm}\right): \delta 172.59,135.70,124.30,122.57,122.02,49.05$, 44.71, 33.76, 31.24, 18.65, 12.78 (Fig. S3).

\section{1-(2-Carboxyethyl)-3-butylimidazolium hexafluorophosphate}


1-(2-Carboxyethyl)-3-butylimidazolium hexafluorophosphate $\left.\left[\mathrm{C}_{4} \mathrm{IMC}_{2} \mathrm{COOH}\right]\left[\mathrm{PF}_{6}\right]\right)$ was prepared by a two-step method. In the first step, $\left[\mathrm{C}_{4} \mathrm{IMC}_{2} \mathrm{COOH}\right] \mathrm{Br}$ was prepared according to the above method. In the second step, $\left[\mathrm{C}_{4} \mathrm{IMC}_{2} \mathrm{COOH}\right] \mathrm{Br}(1.3858 \mathrm{~g}, 5$ mmol) and $\mathrm{NH}_{4} \mathrm{PF}_{6}(0.8965 \mathrm{~g}, 5.5 \mathrm{mmol})$ were dissolved in two portions of deionized water $(5 \mathrm{~mL})$, and stirred at room temperature for $2 \mathrm{~h}$. After decantation of the upper aqueous phase, the crude product was diluted in $30 \mathrm{~mL}$ of methylene chloride and washed with $20 \mathrm{~mL}$ of $\mathrm{H}_{2} \mathrm{O}$, followed by dying over anhydrous $\mathrm{MgSO}_{4}$. The solvent was removed by rotary evaporation, and then dried under vacuum at $60{ }^{\circ} \mathrm{C}$ for $12 \mathrm{~h}$ to obtain a colorless viscous IL. Yield: $\sim 1.47 \mathrm{~g}(85.9 \%)$. $\left[\mathrm{C}_{4} \mathrm{IMC}_{2} \mathrm{COOH}\right]\left[\mathrm{PF}_{6}\right]:{ }^{1} \mathrm{H} \mathrm{NMR}$ (400 MHz, DMSO-d 6 , ppm): $\delta$ 9,39 (s, 1H), $7.84(\mathrm{~s}, 1 \mathrm{H}), 7.71(\mathrm{~s}, 1 \mathrm{H}), 4.37$ (t, 2H), 4.18 (t, 2H), 2.95 (t, 2H), $1.77(\mathrm{~m}, 2 \mathrm{H}), 1.23(\mathrm{~m}, 2 \mathrm{H}), 0.86$ (t, 3H); ${ }^{13} \mathrm{C}$ NMR (100 MHz, DMSO-d $\left.{ }_{6}, \mathrm{ppm}\right): \delta 171.54,136.63,122.44,122.09,48.59,44.72,33.78,31.28,18.76$, 13.21(Fig. S4).

\section{1-Butyl-3-methyl imidazolium hydrogen sulfate}

1-Butyl-3-methyl imidazolium hydrogen sulfate ([BMIM] $\left.\left[\mathrm{HSO}_{4}\right]\right)$ was prepared from chloride salt ([BMIM]Cl) according to literature. ${ }^{2}$ Under nitrogen atmosphere, [BMIM]Cl (3.492 g, $20 \mathrm{mmol})$ was added into a $50 \mathrm{~mL}$ Schlenk bottle at room temperature and dissolved in anhydrous methylene chloride $(10 \mathrm{~mL})$. Then an equivalent amount of concentrated $\mathrm{H}_{2} \mathrm{SO}_{4}(97 \%, 2.022 \mathrm{~g}, 20 \mathrm{mmol})$ was added dropwise to the above solution. The mixture was heated to $40{ }^{\circ} \mathrm{C}$ and refluxed for $48 \mathrm{~h}$. After completion of the reaction, the dichloromethane was removed in a rotary evaporator, while the $\mathrm{HCl}$ by-product formed in the reaction was removed using a vacuum cryogenic device (liquid nitrogen). After further drying at $60{ }^{\circ} \mathrm{C}$ for $12 \mathrm{~h}$, a light pink viscous liquid was obtained. Yield: 4.12 g (87.2\%). [BMIM][HSO $\left.{ }_{4}\right]:{ }^{1} \mathrm{H}$ NMR (400 MHz, DMSO-d 6 , ppm): $\delta 8.50$ (s, 1H), 7.27 (s, 1H), $7.22(\mathrm{~s}, 1 \mathrm{H}), 4.00(\mathrm{t}$, 2H), $3.68(\mathrm{~s}, 3 \mathrm{H}), 1.62(\mathrm{~m}, 2 \mathrm{H}), 1.11(\mathrm{~m}, 2 \mathrm{H}), 0.71(\mathrm{t}, 3 \mathrm{H}) ;{ }^{13} \mathrm{C}$ NMR (100 MHz, DMSO-d 6 , ppm): $\delta 135.99,123.43,122.29,49.10,35.76,31.26,18.40,12.77$. (Fig. S5). 


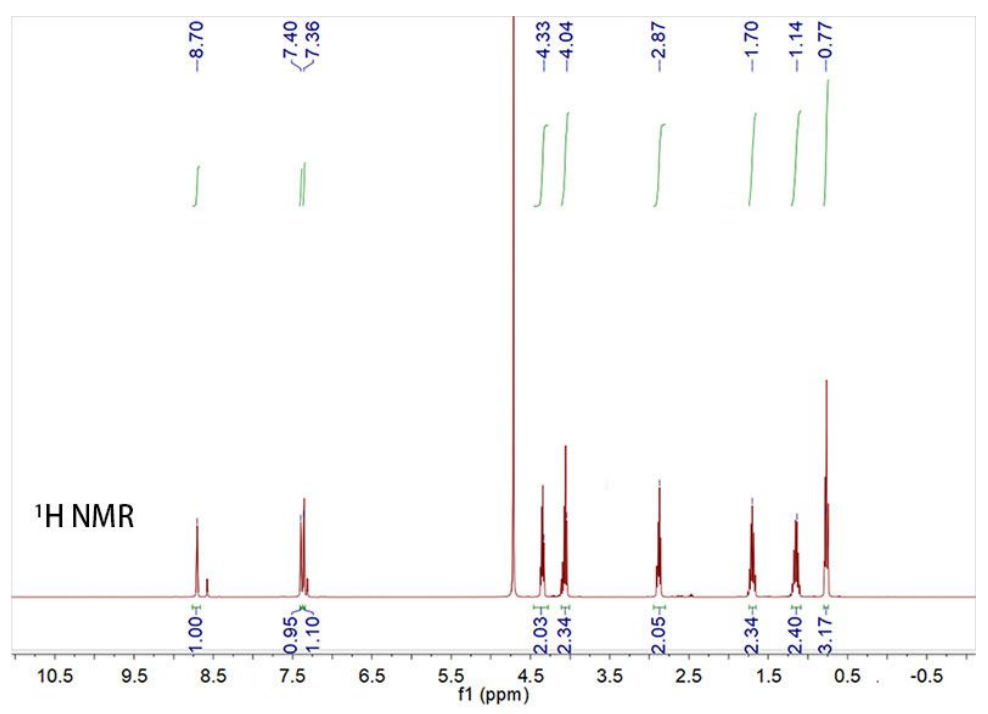

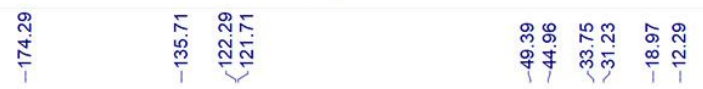

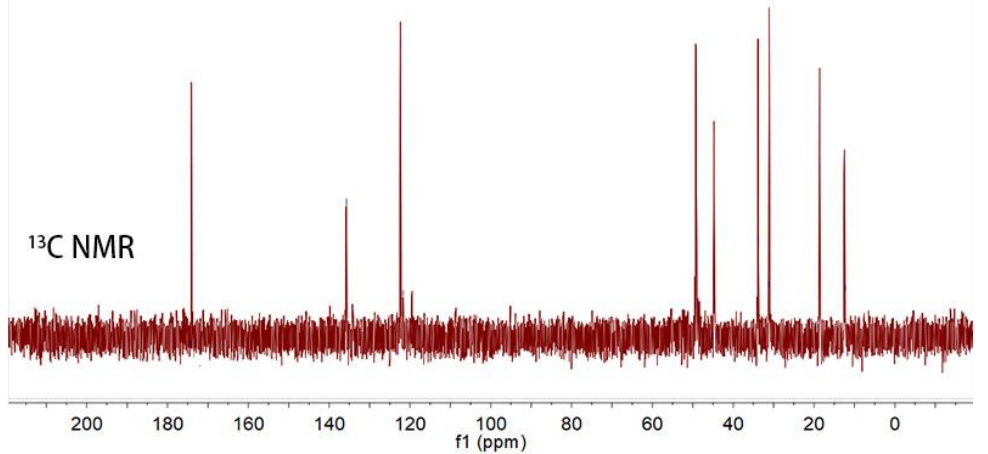

Figure $\mathrm{S} 1{ }^{1} \mathrm{H}$ NMR and ${ }^{13} \mathrm{C}$ NMR spectra of $\left[\mathrm{C}_{4} \mathrm{IMC}_{2} \mathrm{COOH}\right] \mathrm{Br}$. 


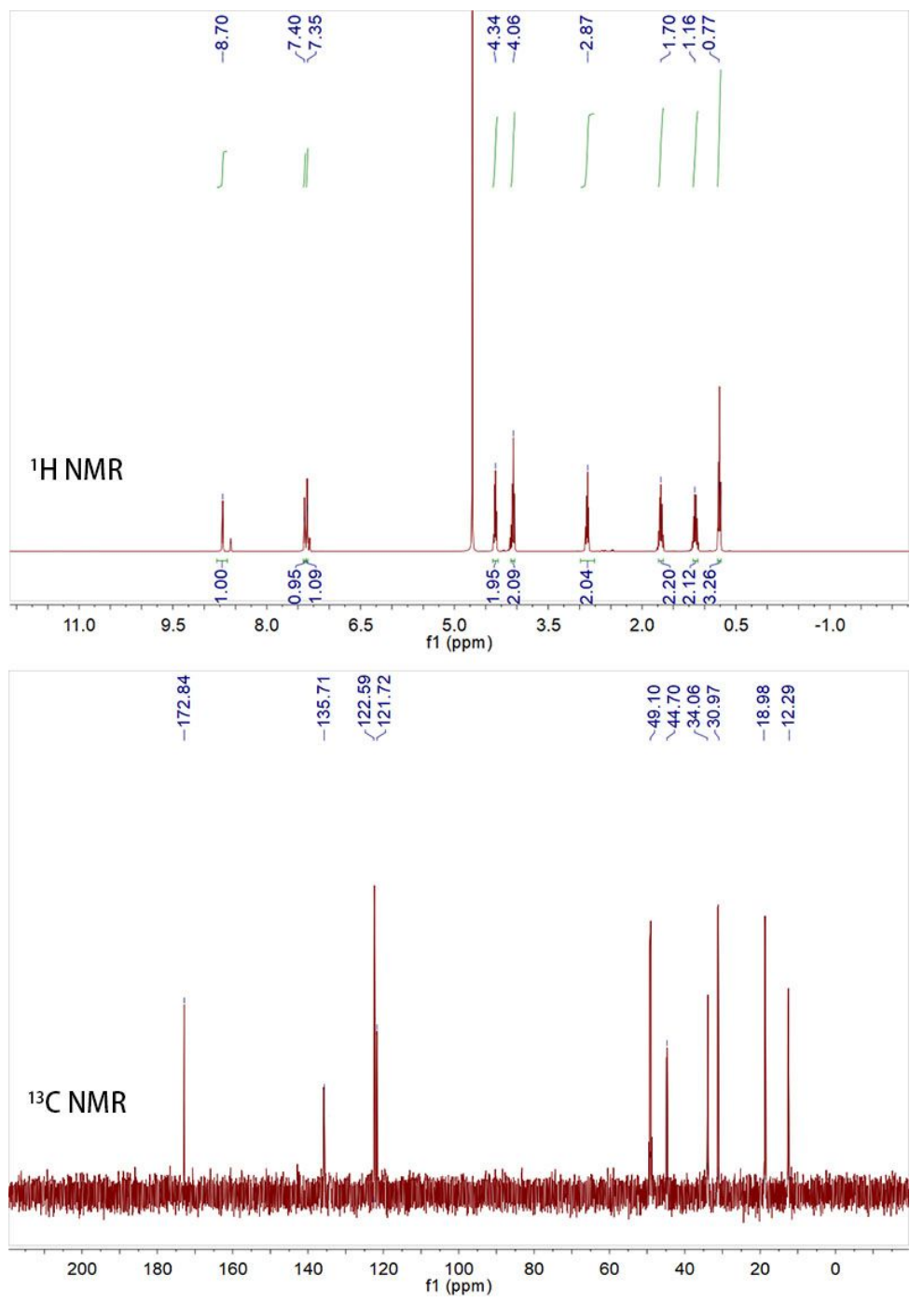

Figure S2 ${ }^{1} \mathrm{H}$ NMR and ${ }^{13} \mathrm{C}$ NMR spectra of $\left[\mathrm{C}_{4} \mathrm{IMC}_{2} \mathrm{COOH}\right]\left[\mathrm{HSO}_{4}\right]$. 

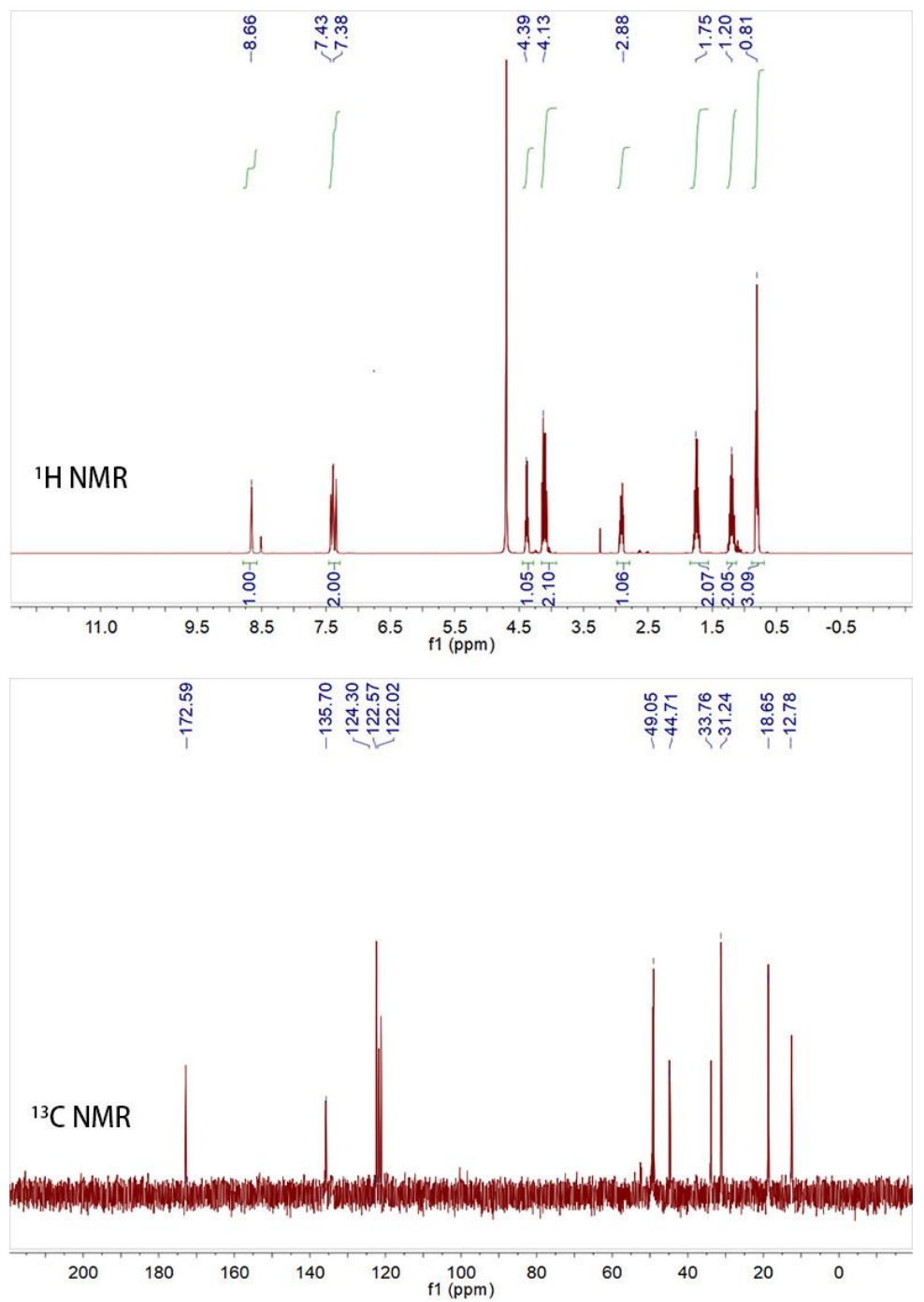

Figure $\mathrm{S} 3{ }^{1} \mathrm{H}$ NMR and ${ }^{13} \mathrm{C}$ NMR spectra of $\left[\mathrm{C}_{4} \mathrm{IMC} \mathrm{M}_{2} \mathrm{COOH}\right][\mathrm{OTf}]$. 


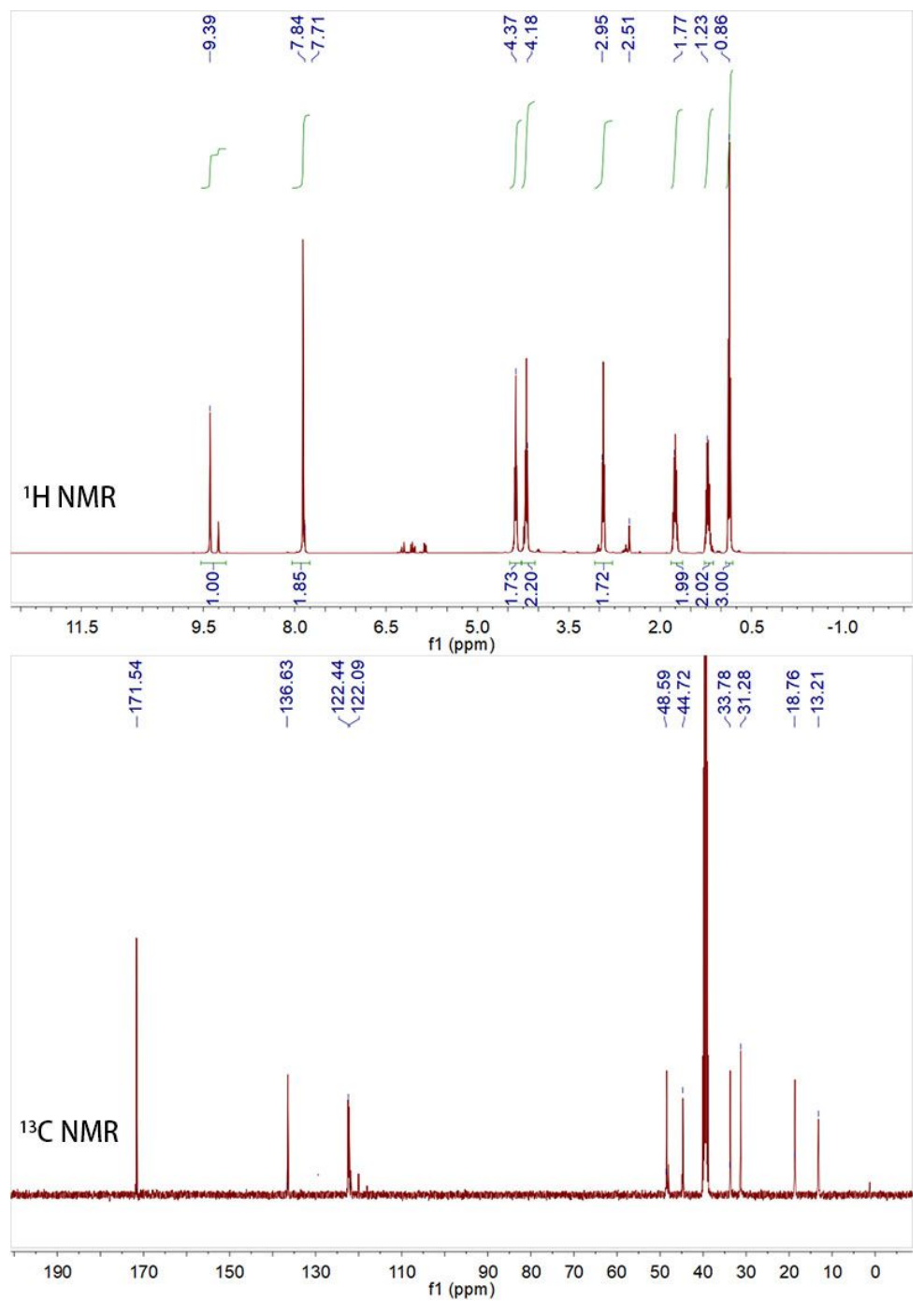

Figure $\mathrm{S} 4{ }^{1} \mathrm{H} \mathrm{NMR}$ and ${ }^{13} \mathrm{C}$ NMR spectra of $\left[\mathrm{C}_{4} \mathrm{IMC}_{2} \mathrm{COOH}\right]\left[\mathrm{PF}_{6}\right]$. 


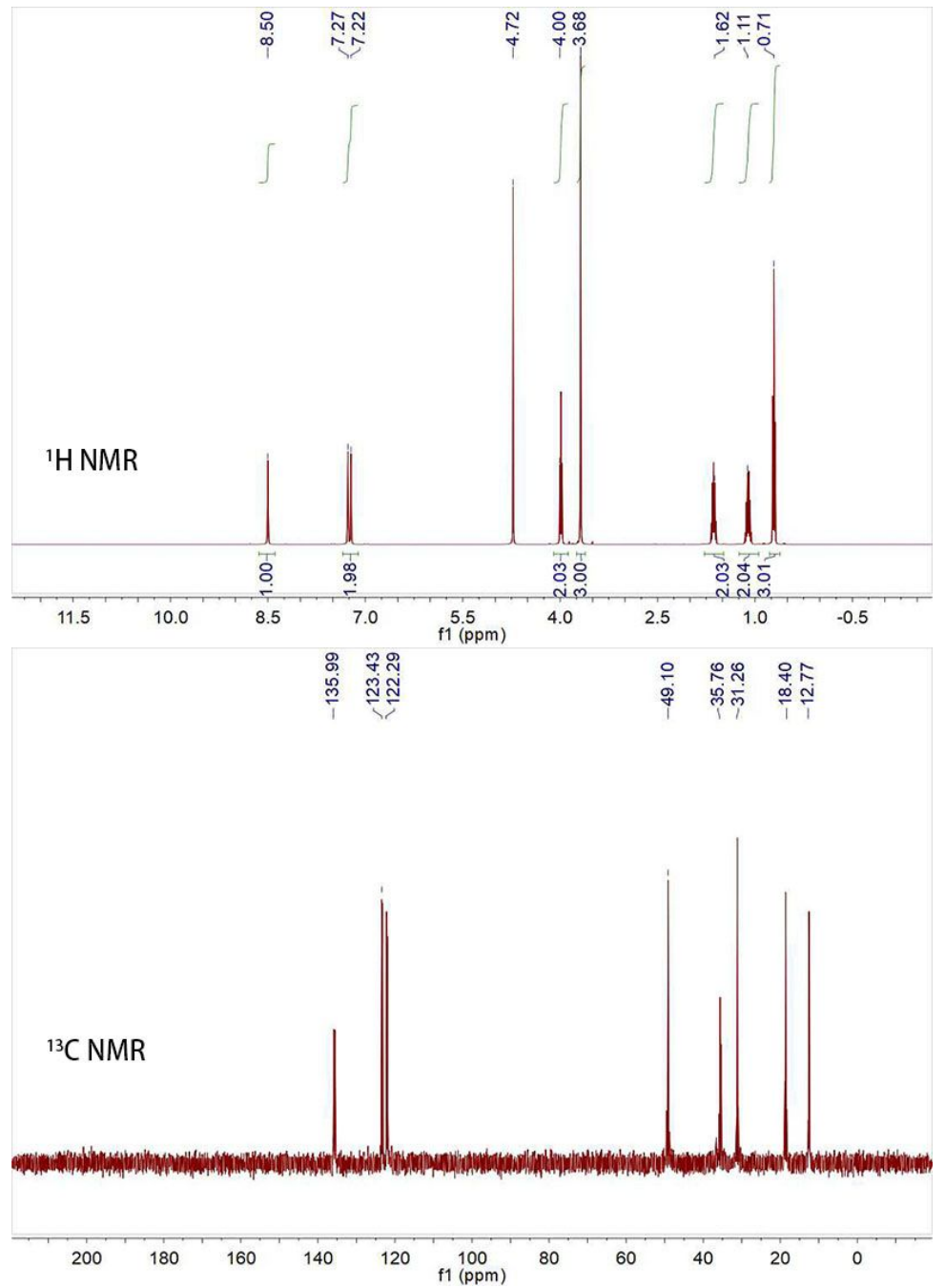

Figure S5 ${ }^{1} \mathrm{H}$ NMR and ${ }^{13} \mathrm{C}$ NMR spectra of [BMIM] $\left[\mathrm{HSO}_{4}\right]$. 


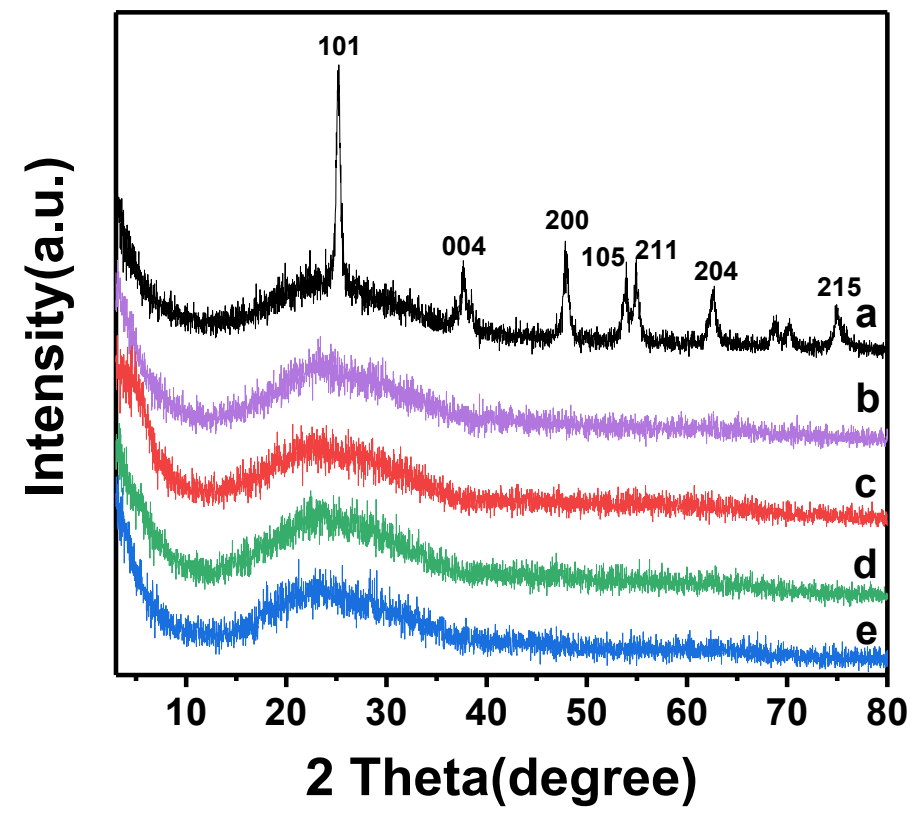

Figure S6 XRD patterns of a)500- $\mathrm{TiO}_{2}$ and different IL-stabilized Ti oxoclusters; b) Ti oxo-Br; c) Ti oxo-HSO 4 ; d) Ti oxo-OTf; e) Ti oxo-PF 6 . 

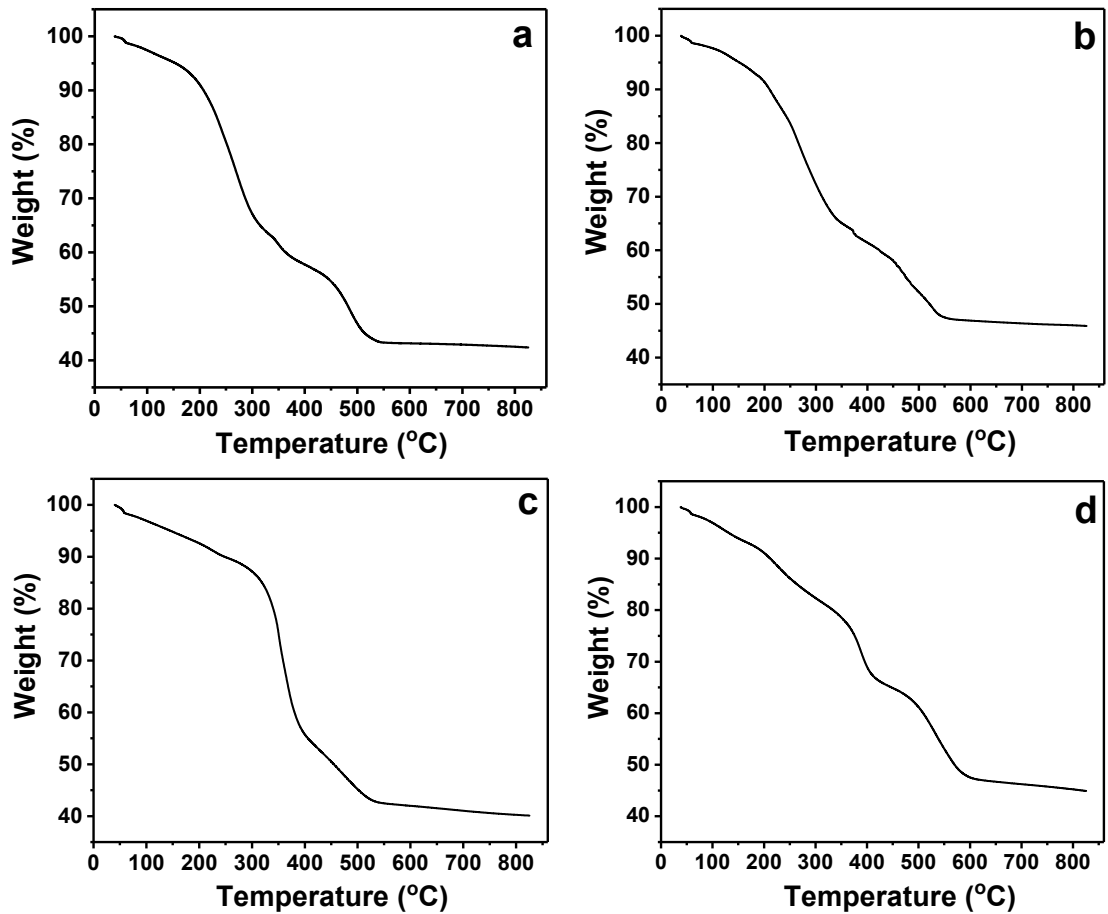

Figure S7 TGA patterns of different Ti-oxo clusters. a) Ti oxo-Br; b) Ti oxo-HSO 4 ; c) Ti oxo-OTf; d) Ti oxo-PF 6 . 


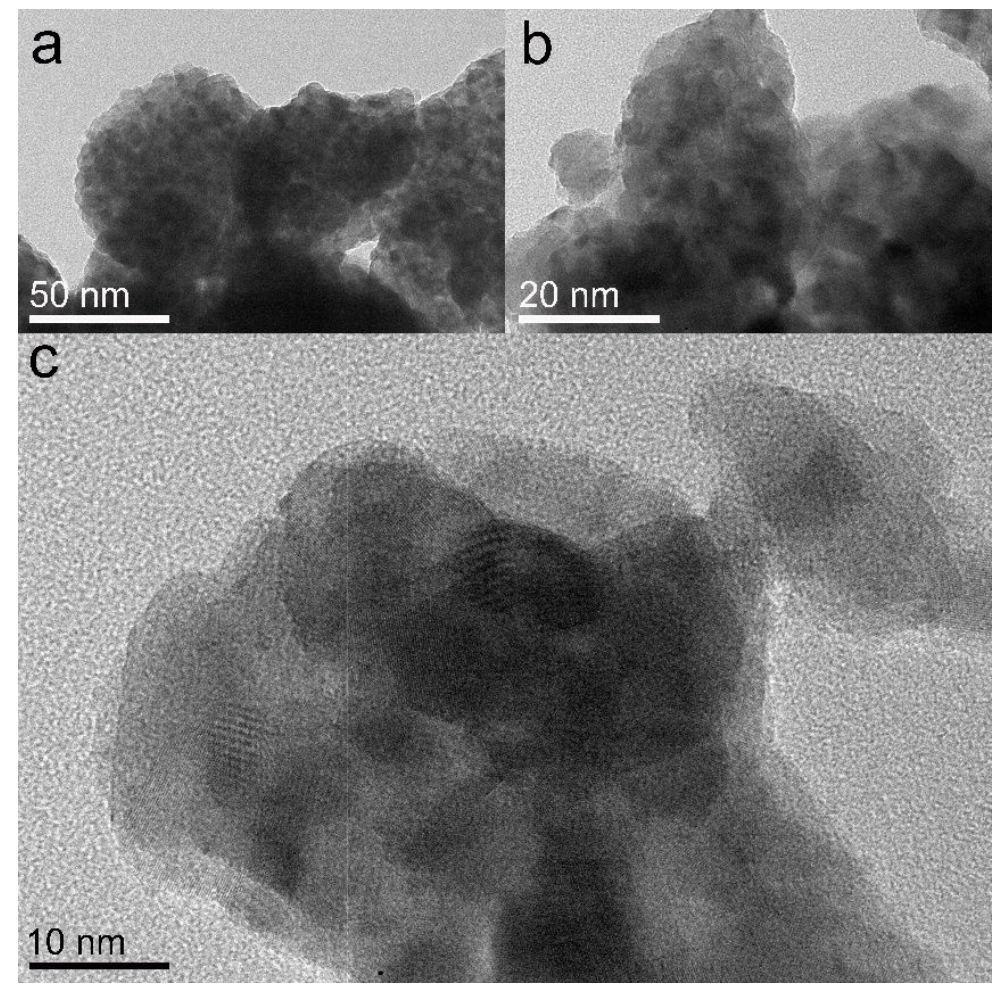

Figure S8 HRTEM images of 500- $\mathrm{TiO}_{2}(\mathrm{a}, \mathrm{b}, \mathrm{c})$ with different magnification. 


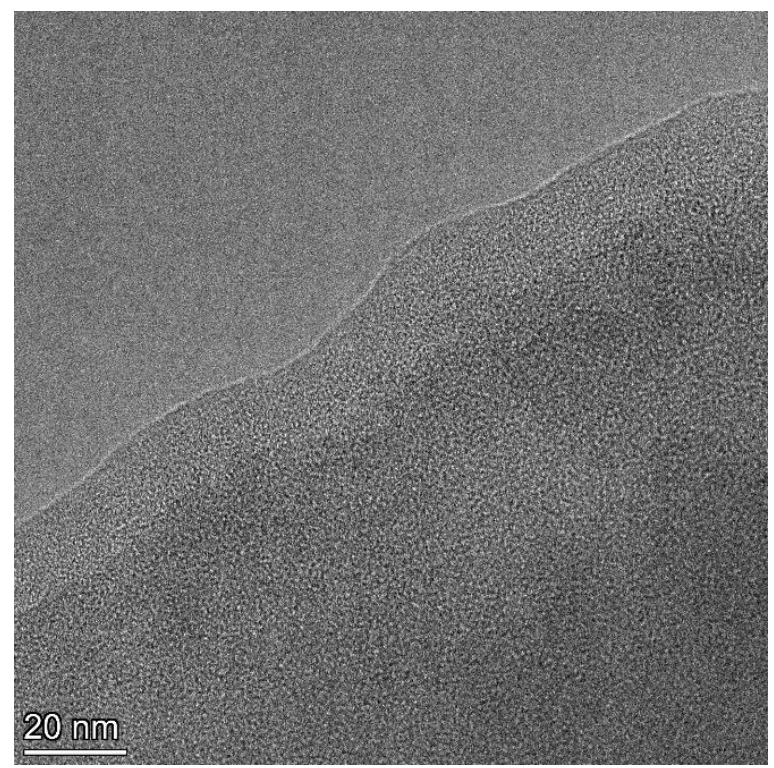

Figure S9 HRTEM image of Ti oxo-[BMIM] $\mathrm{HSO}_{4}$ catalyst. 


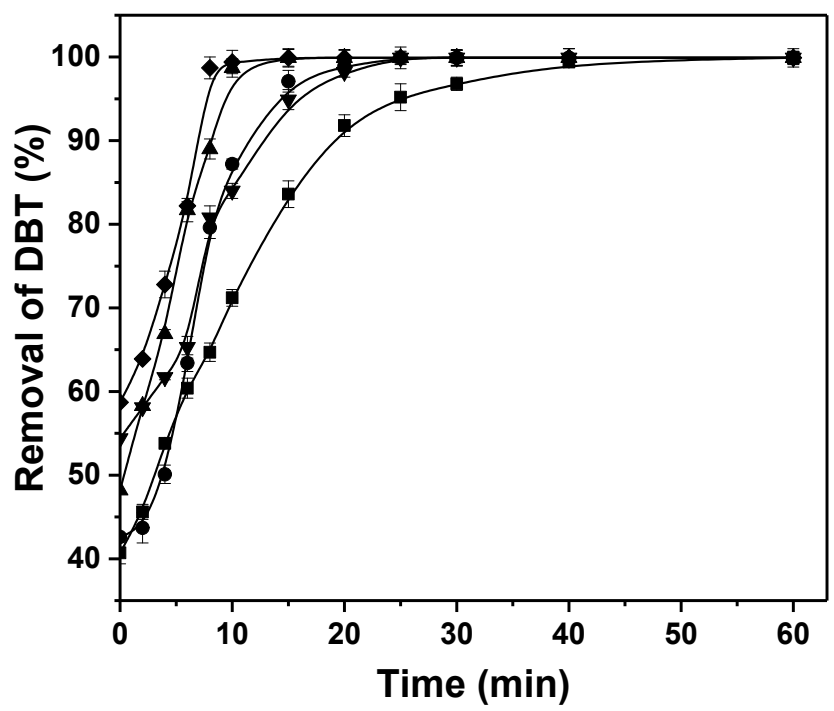

Figure S10 Removal of DBT versus the reaction time with different volume ratios of

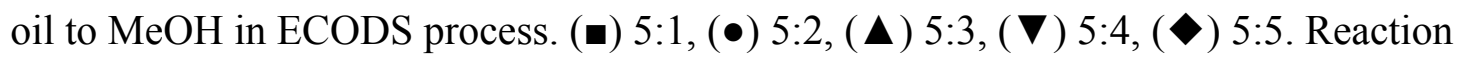
conditions: DBT (S content of $3000 \mathrm{ppm}$ ) in n-octane $(5 \mathrm{~mL}), \mathrm{H}_{2} \mathrm{O}_{2}$ (3 equiv., $30 \%$ aqueous solution), $\mathrm{Ti}$ oxo- $\mathrm{HSO}_{4}(10 \mathrm{mg}), \mathrm{T}=60^{\circ} \mathrm{C}$. 


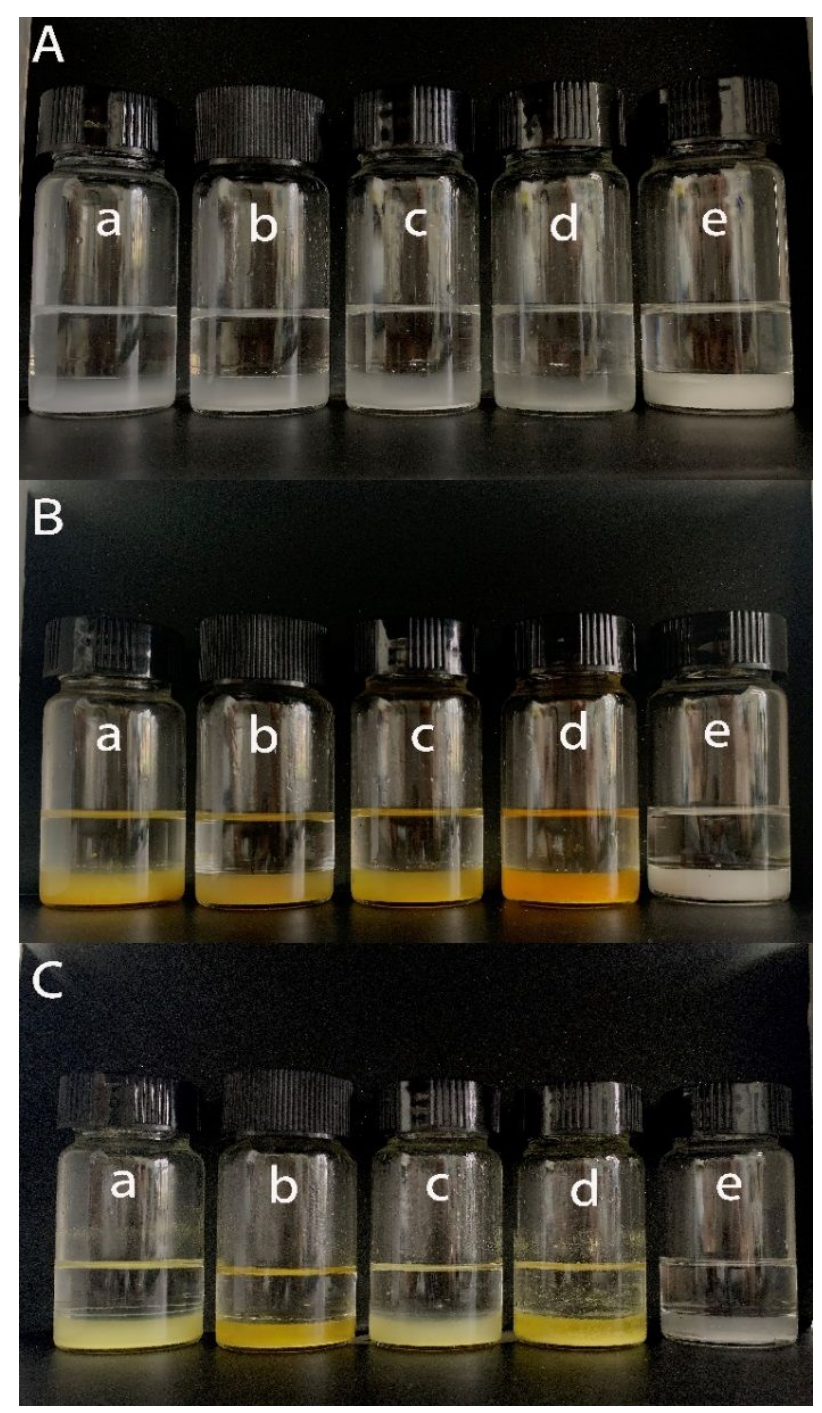

Figure S11 (A) The photograph of the IL-stabilized Ti oxoclusters and 500- $\mathrm{TiO}_{2}$ catalysts in methanol/model oil of DBT biphasic systems; (B) After $\mathrm{H}_{2} \mathrm{O}_{2}$ was introduced into the mixture to form ECODS system; (C) Reaction for $30 \mathrm{~min}$. From left to right: a) Ti oxo-Br; b) Ti oxo- $\mathrm{HSO}_{4}$; c) Ti oxo-OTf; d) Ti oxo-PF 6 ; e) 500- $\mathrm{TiO}_{2}$. Conditions: DBT (S content of $3000 \mathrm{ppm}$ ) in n-octane $(5 \mathrm{~mL}), \mathrm{H}_{2} \mathrm{O}_{2}$ (3 equiv., $30 \%$ aqueous solution), Catalyst: $50 \mathrm{mg}, \mathrm{T}=60{ }^{\circ} \mathrm{C}, \mathrm{CH}_{3} \mathrm{OH}(2.0 \mathrm{~mL})$. 


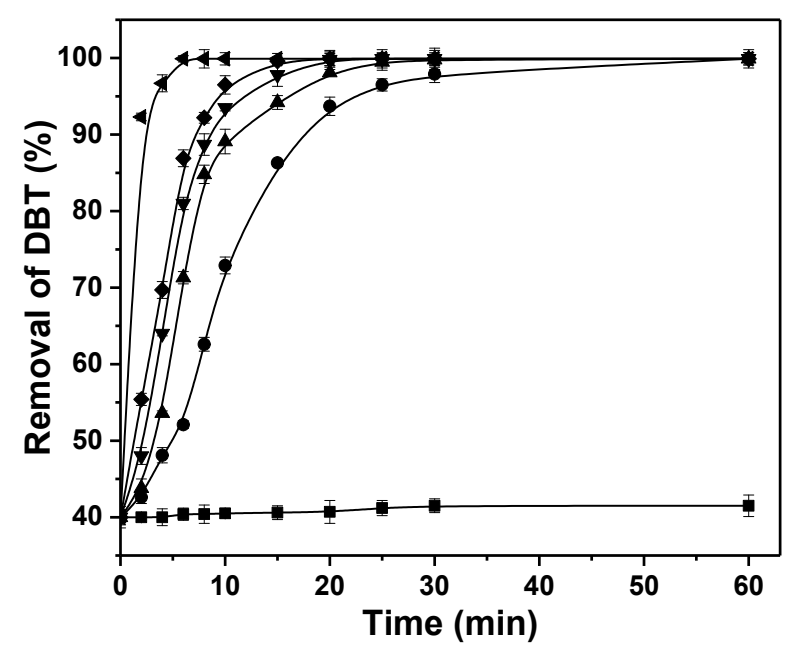

Figure S12 Removal of DBT versus the reaction time with different amounts of Ti-

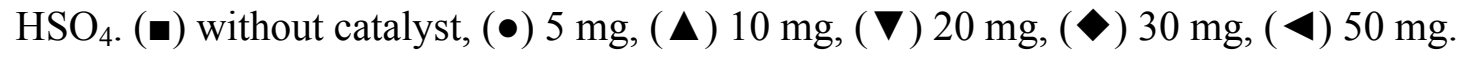
Reaction conditions: DBT (S content of $3000 \mathrm{ppm}$ ) in n-octane $(5 \mathrm{~mL}), \mathrm{H}_{2} \mathrm{O}_{2}$ (3equiv., $30 \%$ aqueous solution), Catalyst: $\mathrm{Ti}$ oxo- $\mathrm{HSO}_{4}, \mathrm{~T}=60{ }^{\circ} \mathrm{C}, \mathrm{CH}_{3} \mathrm{OH}(2.0 \mathrm{~mL})$. 


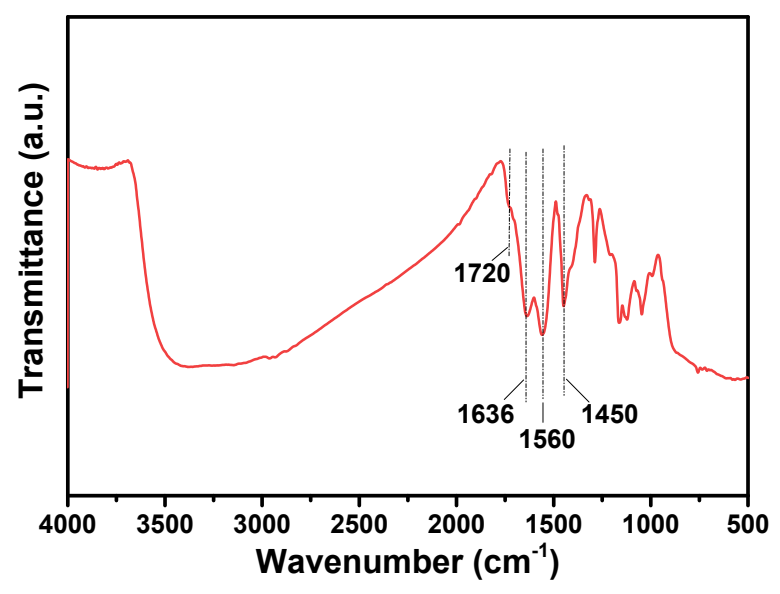

Figure S13 FT-IR spectra of the spent Ti oxo- $\mathrm{HSO}_{4}$ catalyst (without dryness) after six catalytic recycles. 


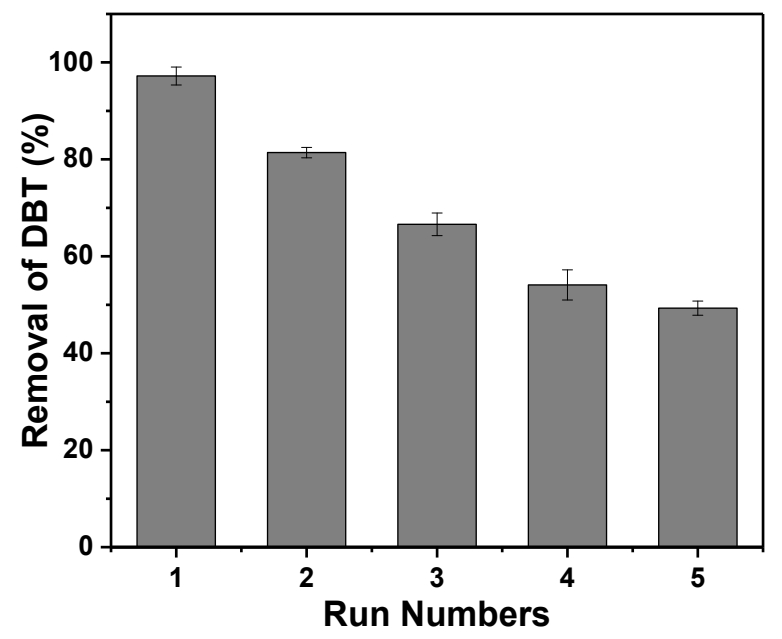

Figure S14 Reusability of Ti oxo-[BMIM] $\mathrm{HSO}_{4}$ catalyst. Reaction conditions for ECODS process: DBT (S content of $3000 \mathrm{ppm}$ ) in n-octane $(5 \mathrm{~mL}), \mathrm{H}_{2} \mathrm{O}_{2}$ (3equiv., 30\% aqueous solution), Ti oxo-[BMIM] $\mathrm{HSO}_{4}(50 \mathrm{mg}), \mathrm{T}=60{ }^{\circ} \mathrm{C}, \mathrm{CH}_{3} \mathrm{OH}(2.0 \mathrm{~mL})$. 


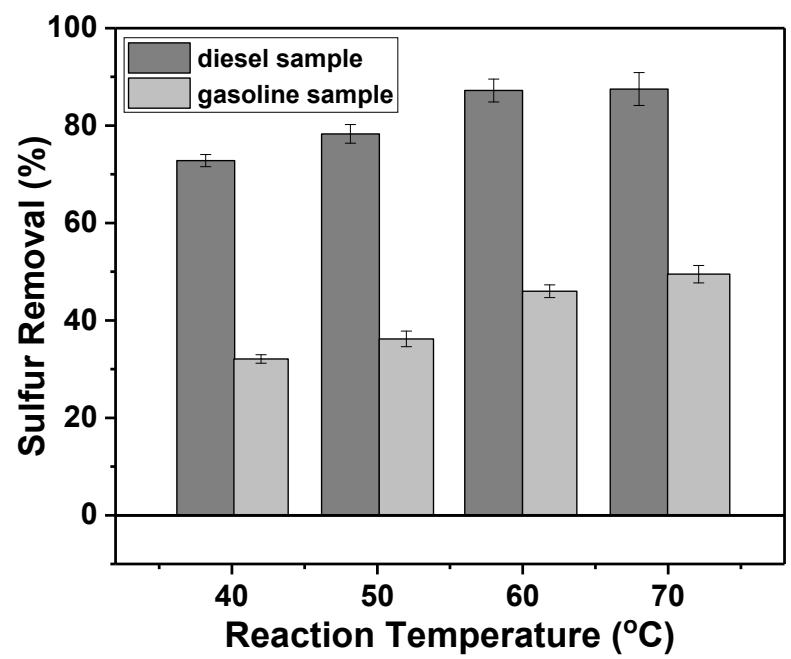

Figure S15 Effect of temperature on sulfur removal of actual fuel samples (diesel or gasoline). Reaction conditions: Diesel sample (20.0 mL), $\mathrm{H}_{2} \mathrm{O}_{2}$ (6 equiv., $30 \%$ aqueous solution), Ti oxo- $\mathrm{HSO}_{4}(100 \mathrm{mg}), \mathrm{CH}_{3} \mathrm{OH}(10.0 \mathrm{~mL}), 2 \mathrm{~h}$; Gasoline sample (20.0 mL), $\mathrm{H}_{2} \mathrm{O}_{2}$ (6 equiv., $30 \%$ aqueous solution), Ti oxo- $\mathrm{HSO}_{4}(100 \mathrm{mg}), \mathrm{CH}_{3} \mathrm{OH}(10.0 \mathrm{~mL}), 12$ h. 

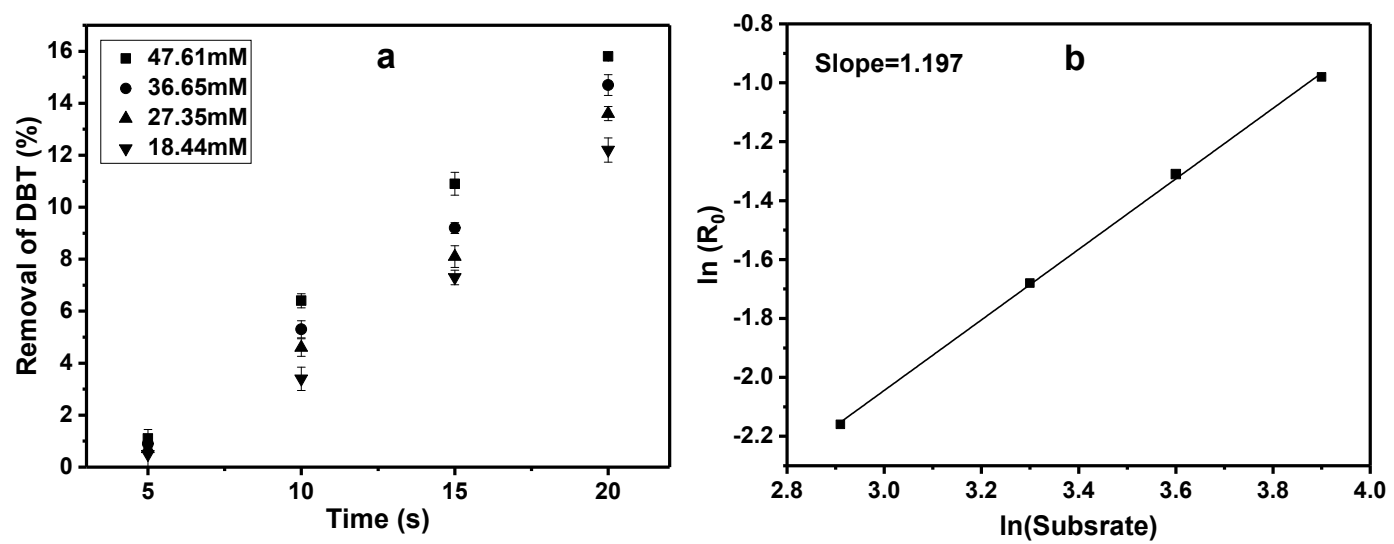

Figure S16 The relationship between the reaction rate $\left(\mathrm{R}_{0}\right)$ and the concentration of $\operatorname{DBT}(\mathrm{a}, \mathrm{b})$. Reaction conditions for a and b: DBT (18.44 mM-47.61 mM), $\mathrm{H}_{2} \mathrm{O}_{2}(0.29$ M), Ti oxo- $\mathrm{HSO}_{4}$ (ca. $10 \mathrm{mg}$ ), $\mathrm{T}=40{ }^{\circ} \mathrm{C}$. All reaction profiles have been attained at low conversions $(<20 \%)$. 
Table S1 Comparison of catalytic systems for oxidative desulfurization of DBT in the presence of $\mathrm{H}_{2} \mathrm{O}_{2}$

\begin{tabular}{|c|c|c|c|c|c|c|c|}
\hline Entry & Catalysts & Extractants & $\begin{array}{c}\mathrm{T} \\
{ }^{\circ} \mathrm{C}\end{array}$ & $\begin{array}{c}\mathrm{t}^{\mathrm{a}} \\
/ \mathrm{min}\end{array}$ & $\begin{array}{l}\mathrm{H}_{2} \mathrm{O}_{2} / \\
\mathrm{Sub}^{\mathrm{b}}\end{array}$ & $\begin{array}{c}\text { S Removal } \\
\quad / \%\end{array}$ & Ref. \\
\hline 1 & $\mathrm{TBAPMo}_{11} \mathrm{Cu} @ \mathrm{CuO}^{\mathrm{c}}$ & None & 35 & 60 & $6: 1$ & 98 & 3 \\
\hline 2 & $\mathrm{Ti}_{32}-\mathrm{BTA}^{\mathrm{d}}$ & None & 60 & 60 & $6: 1$ & 100 & 4 \\
\hline 3 & $\mathrm{MTO}^{\mathrm{e}}$ & {$[\mathrm{Bmim}] \mathrm{BF}_{4}{ }^{\mathrm{f}}$} & 60 & 180 & $6: 1$ & 99 & 5 \\
\hline 4 & $150-\mathrm{TiO}_{2}^{\mathrm{g}}$ & {$[\mathrm{Bmim}] \mathrm{BF}_{4}$} & 40 & 90 & $3: 1$ & 99.1 & 6 \\
\hline 5 & Anhydrous $\mathrm{FeCl}_{3}$ & {$[\mathrm{Bmim}] \mathrm{BF}_{4}$} & 30 & 240 & $6: 1$ & 96.1 & 7 \\
\hline 6 & $\mathrm{C}_{16} \mathrm{PW} / \mathrm{TiO}_{2}{ }^{\mathrm{h}}$ & {$[\mathrm{Bmim}] \mathrm{BF}_{4}$} & 60 & 60 & $3: 1$ & 100 & 8 \\
\hline 7 & {$[\mathrm{Bmim}] \mathrm{FeCl}_{4} / \mathrm{SiO}_{2}{ }^{\mathrm{i}}$} & {$[\mathrm{Omim}] \mathrm{BF}_{4}{ }^{\mathrm{j}}$} & 30 & 60 & $4: 1$ & 97.3 & 9 \\
\hline 8 & {$\left[\mathrm{C}_{2}(\mathrm{mim})_{2}\right] \mathrm{PW}_{12} \mathrm{O}_{40}$} & $\mathrm{MeCN}$ & 50 & 60 & $6: 1$ & 98.4 & 10 \\
\hline 9 & $\mathrm{VOHPO}_{4} \cdot 5 \mathrm{H}_{2} \mathrm{O} / \mathrm{KIT}-6^{\mathrm{k}}$ & $\mathrm{MeCN}$ & 40 & 60 & $7: 1$ & 97.7 & 11 \\
\hline 10 & MIL-125 & Methanol & 60 & 120 & $8: 1$ & 99.9 & 12 \\
\hline 11 & $25 \mathrm{Mo}^{-\mathrm{Al}} \mathrm{l}^{\mathrm{m}}$ & $\mathrm{MeCN}$ & 60 & 30 & $2.3: 1$ & 100 & 13 \\
\hline 12 & HP-UiO-66 ${ }^{\mathrm{n}}$ & $\mathrm{MeCN}$ & 60 & 30 & $4: 1$ & 100 & 14 \\
\hline 13 & HPMo/NA-SBA-15 & None & 80 & 5 & $8: 1$ & 99.9 & 15 \\
\hline 14 & $\mathrm{Ti}$ oxo- $-\mathrm{HSO}_{4}^{\mathrm{p}}$ & Methanol & 60 & 30 & $3: 1$ & 99.9 & this \\
\hline 15 & $\mathrm{Ti}$ oxo- $\mathrm{HSO}_{4}{ }^{\mathrm{q}}$ & Methanol & 60 & 5 & $3: 1$ & 99.9 & $\begin{array}{l}\text { work } \\
\text { this } \\
\text { work }\end{array}$ \\
\hline
\end{tabular}

${ }^{a}$ t: reaction time of oxidation process; ${ }^{b} \mathrm{H}_{2} \mathrm{O}_{2} / \mathrm{Sub}$ : The molar ratio of $\mathrm{H}_{2} \mathrm{O}_{2}$ to DBT; 'TBA: tetrabutylammonium bromide; ${ }^{\mathrm{d}} \mathrm{Ti}_{32}$-BTA: $\mathrm{Ti}_{32}$-oxo macrocycles functionalized with butyrate ligands; ${ }^{\mathrm{e} M T O}$ methyltrioxorhenium; ${ }^{\mathrm{f} B m i m: ~ 1-B u t y l-3-}$ methylimidazolium cation; ${ }^{\mathrm{g}} 150-\mathrm{TiO}_{2}$ : titanium dioxide calcined at $150{ }^{\circ} \mathrm{C} ;{ }^{\mathrm{h}} \mathrm{C}_{16} \mathrm{PW}$ : $\left[\mathrm{C}_{16} \mathrm{mim}\right]_{3} \mathrm{PW}_{12} \mathrm{O}_{40} ;{ }^{i} \mathrm{SiO}_{2}$ : silica gel. jOmim: 1-octyl-3-methylimidazolium cation; ${ }^{\mathrm{k} K I T-6:}$ cubic mesoporous silica; ${ }^{1} \mathrm{MIL}-125$ : Ti-containing MOF; ${ }^{\mathrm{m}} 25 \mathrm{Mo}-\mathrm{Al}$ : 25\% $\mathrm{MoO}_{3}-\mathrm{Al}_{2} \mathrm{O}_{3} ;{ }^{\mathrm{n}} \mathrm{HP}-\mathrm{UiO}-66$ : hierarchically porous zirconium-based MOF; ${ }^{\circ} \mathrm{HPMo} / \mathrm{NA}$ : 12-phosphomolybdic acid/nicotinic acid; ${ }^{\mathrm{p}} \mathrm{m}\left(\mathrm{Ti}\right.$ oxo- $\left.\mathrm{HSO}_{4}\right)=10 \mathrm{mg} ;{ }^{\mathrm{q}} \mathrm{m}$ (Ti oxo$\left.\mathrm{HSO}_{4}\right)=50 \mathrm{mg}$. 

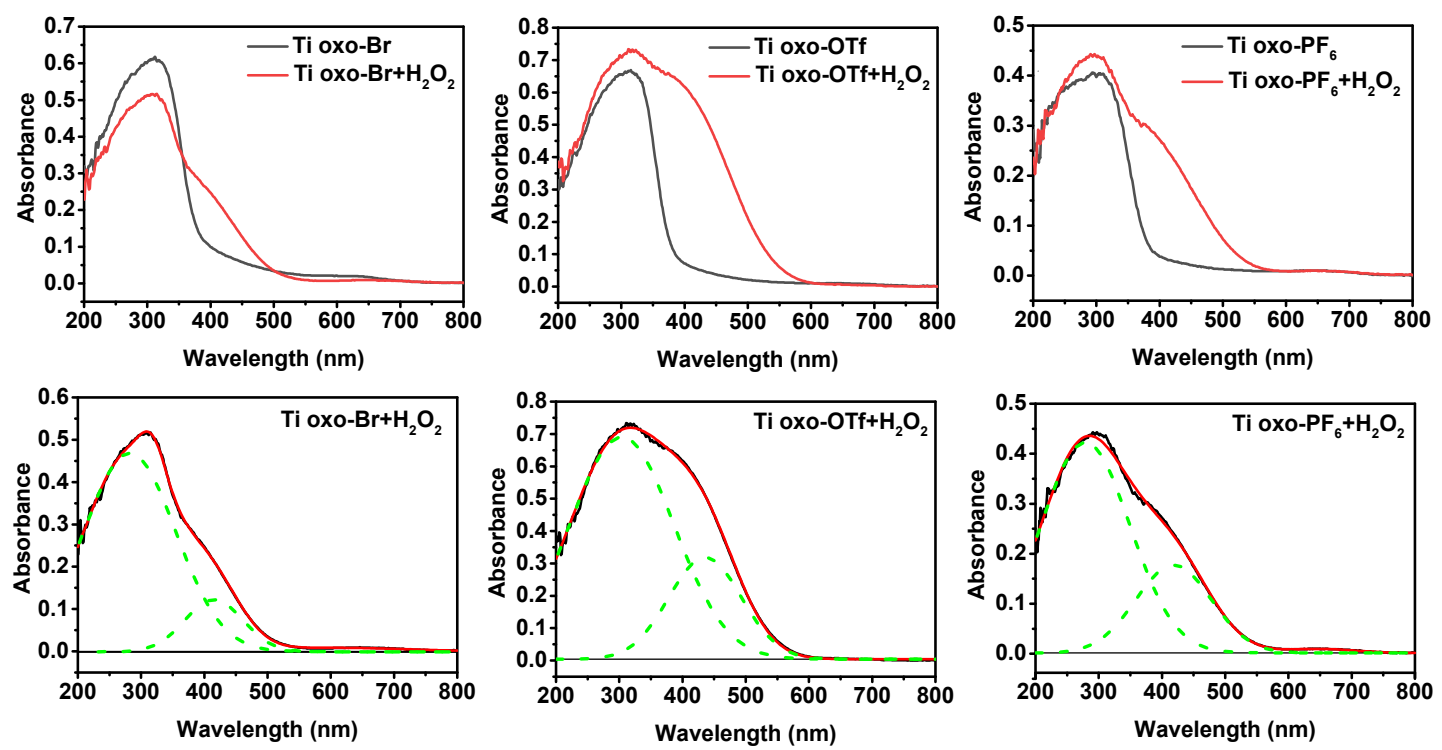

Figure S17 UV Diffuse Reflectance Spectrum of Ti oxoclusters (Row 1, Black line) and $\mathrm{Ti}$ oxoclusters treated with $\mathrm{H}_{2} \mathrm{O}_{2}$ (Row 1, Red line) and the corresponding deconvoluted spectra of Ti oxoclusters treated with $\mathrm{H}_{2} \mathrm{O}_{2}$ (Row 2). 


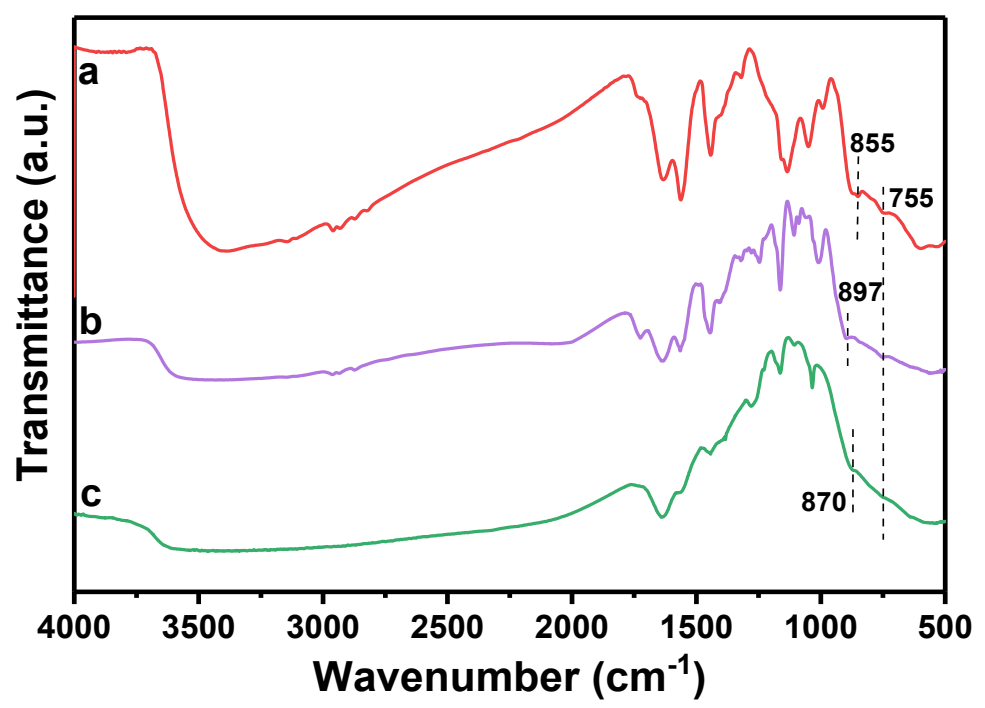

Figure S18 FT-IR spectra of Ti oxoclusters treated with $\mathrm{H}_{2} \mathrm{O}_{2}$ : a) Ti oxo- $\mathrm{HSO}_{4}$, b) Ti oxo-OTf, c) Ti oxo-PF 6 .

\section{References}

(1) Han, L. N.; Choi, S. J.; Park, M. S.; Lee, S. M.; Kim, Y. J.; Kim, M. I.; Liu, B. Y.; Park, D. W. Carboxylic acid functionalized imidazolium-based ionic liquids: efficient catalysts for cycloaddition of $\mathrm{CO}_{2}$ and epoxides. Reac. Kinet. Mech. Cat. 2012, 106, 25-35.

(2) Tajika, H.; Niknam, K.; Parsa, F. Using Acidic Ionic Liquid 1-Butyl-3methylimidazolium Hydrogen Sulfate in Selective Nitration of Phenols under Mild Conditions. J. Iran. Chem. Soc. 2009, 6, 159-164.

(3) Aghbolagh, Z. S.; Khanmohammadi Khorrami M. R.; Rahmatyan, M. S. Oxidative Desulfurization and Denitrogenation of Simulated Fuels Catalyzed by $\mathrm{TBAPMo}_{11} \mathrm{Cu} @ \mathrm{CuO}$ as a High-Performance and Recoverable Heterogeneous PhaseTransfer Catalyst. Energy Fuels 2020, 34, 16366-16380.

(4) Lv, H. T.; Cui, Y.; Zou, G. D.; Li, N.; Yang, P.; Fan, Y. Synthesis of titanium-oxo macrocyles and their catalytic properties for oxidative desulfurization. Dalton Trans. 2019, 48, 14044-14048.

(5) Zhou, M.; Meng, W.; Li, Y.; Wang, Q.; Li, X.; Zang, S. Extractive and Catalytic Oxidative Desulfurization of Gasoline by Methyltrioxorhenium in Ionic Liquids. 
Energy Fuels 2013, 28, 516-521.

(6) Zheng, D.; Zhu, W.; Xun, S.; Zhou, M.; Zhang, M.; Jiang, W.; Qin, Y.; Li, H. Deep oxidative desulfurization of dibenzothiophene using low-temperature-mediated titanium dioxide catalyst in ionic liquids. Fuel 2015, 159, 446-453.

(7) Zhang, J.; Zhu, W.; Li, H.; Jiang, W.; Jiang, Y.; Huang, W.; Yan, Y. Deep oxidative desulfurization of fuels by Fenton-like reagent in ionic liquids. Green Chem. 2009, 11, 1801-1807.

(8) Xun, S. H.; Yu, Z. H.; He, M. Q.; Wei, Y. C.; Li, X. W.; Zhang, M.; Zhu, W. S.;

Li, H. M. Supported phosphotungstic-based ionic liquid as an heterogeneous catalyst used in the extractive coupled catalytic oxidative desulfurization in diesel. Res. Chem. Intermediat. 2019, 45, 4315-4334.

(9) Xun, S.; Zhu, W. S.; Zheng, D.; Zhang, L.; Liu, H.; Yin, S.; Zhang, M.; Li, H. M. Synthesis of metal-based ionic liquid supported catalyst and its application in catalytic oxidative desulfurization of fuels. Fuel 2014, 136, 358-365.

(10)Li, J. W.; Guo, Y. W.; Tan, J. J.; Hu, B. Polyoxometalate Dicationic Ionic Liquids as Catalyst for Extractive Coupled Catalytic Oxidative Desulfurization. Catalysts 2021, 11, No. 356.

(11)Moslemi, A.; Chermahini, A. N.; Sarpiri, J. N.; Rezaei, S.; Barati, M. $\mathrm{VOHPO}_{4} \cdot 5 \mathrm{H}_{2} \mathrm{O} / \mathrm{KIT}-6$ composites: Preparation and their application in extractive and catalytic oxidation desulfurization of benzothiophene and dibenzothiphene. J. Taiwan Inst. Chem. E. 2019, 97, 237-246.

(12)Akopyan, A. V.; Shlenova, A. O.; Cherednichenko, K. A.; Polikarpova, P. D. Immobilized Multifunctional Ionic Liquids for Highly Efficient Oxidation of SulfurContaining Compounds in Model Fuels. Energy Fuels 2021, 35, 6755-6764.

(13)Huang, H.; Zhou, Z. W.; Qin, J.; Li, Y.; Liu, G.; Wu, W. L. A facile synthesis of mesoporous Mo-Al oxides for desulfurization of dibenzothiophene in the extractive catalytic oxidative desulfurization system. React. Kinet. Mech. Catal. 2020, 130, 363379.

(14)Hao, L. D.; Stoian, S. A.; Weddle, L. R.; Zhang, Q. Zr-Based MOFs for oxidative desulfurization: what matters? Green Chem. 2020, 22, 6351-6356. 
(15)Akopyan, A. V.; Shlenova, A. O.; Cherednichenko, K. A.; Polikarpova, P. D. Immobilized Multifunctional Ionic Liquids for Highly Efficient Oxidation of SulfurContaining Compounds in Model Fuels. Energy Fuels 2021, 35, 6755-6764. 